
\title{
28 Research Square \\ Learning burnout: evaluating the role of social support in medical students
}

\section{Jiayu Zhang}

Huazhong University of Science and Technology https://orcid.org/0000-0001-6977-4977

\section{Tao Shu}

Huazhong University of Science and Technology

\section{Ming Xiang}

Huazhong University of Science and Technology

\section{Zhanchun Feng ( $\nabla$ zcfeng@hust.edu.cn)}

Huazhong University of Science and Technology Wuhan https://orcid.org/0000-0001-6021-0016

\section{Research article}

Keywords: Learning burnout, Social support, Medical students

Posted Date: October 26th, 2020

DOI: https://doi.org/10.21203/rs.3.rs-53416/v2

License: (9) This work is licensed under a Creative Commons Attribution 4.0 International License. Read Full License 


\section{Abstract}

\section{Background:}

Burnout is a stress-induced syndrome that is considered closely related to work. Although social support could reduce burnout syndrome, the effect of it on learning burnout in medical students remains unclear. The objectives of the study are to evaluate the association between learning burnout and social support in a cohort of Chinese medical students.

\section{Methods:}

A cross-sectional online survey was distributed to students who participated in online learning in a medical college in Wuhan during the COVID-19 epidemic. We used the Lian version of the Maslach Burnout Inventory (MBI) to assess learning burnout and the Social Support Rating Scale (SSRS) to assess social support.

\section{Results:}

A total of 684 students completed the survey (response rate of $30.9 \%$ ), of which $315(46.12 \%)$ met standard criteria for learning burnout. We found grade, family income, learning time, and the number of interactions with teachers or classmates had an effect on students' learning burnout. After adjusting for the grade and residence, there was a significant and relevant association between the social support and learning burnout. (adjusted odds ratio, 0.93 for 1-point decrease in total SSRS score; $95 \% \mathrm{Cl}, 0.90-0.96$; $\mathrm{p}<0.001)$.

\section{Conclusions:}

Learning burnout was highly prevalent in medical students of our college. The social support especially subjective support and utilization of support played a protective role in reducing the risk of learning burnout.

\section{Practice Points}

Social support, especially subjective support and utilization of support had a positive effect on alleviating learning burnout.

\section{Background}

Burnout is a state of psychological distress, which is widely considered an important work-related syndrome. Research on burnout has been done based on the sample of practicing physicians in medical fields, however, more and more researchers are recently beginning to turn their attention to college students. Medical students could also be affected by burnout. The burnout is called learning burnout or academic burnout, which both originate from the definition of burnout and contain the same core 
elements ${ }^{[1,2]}$. Learning burnout is defined as a combination of emotional exhaustion, cynicism and academic inefficacy that cause by their incapacity to meet academic requirements ${ }^{[3,4]}$.

Learning burnout has a higher prevalence among medical students. A systematic review reported $25.8 \%$ to $52.1 \%$ of medical students had above moderate levels of burnout in China ${ }^{[5]}$. The wide range of burnout levels may be explained by the use of different definitions, measurements and study designs. Moreover, learning burnout has serious negative effects on students and the quality of health care services. It could undermine medical students' professional development, diminish personal and professional qualities (e.g. honesty, integrity, altruism, self-regulation) ${ }^{[6-8]}$, and cause problems such as increased medical errors, reduced quality of patient care, and low patient satisfaction ${ }^{[9,10]}$. Furthermore, some demographic variations may result in differences in learning burnout, such as gender, grade, family economic aspects, employment status ${ }^{[11,12]}$. However, all studies have focused on burnout in the form of learning at school, but not online learning at home.

Social support is defined as assistance and protection provided by others through formal or informal means ${ }^{[13]}$. The absence of social support is considered to be one of the main stress-inducing factors, a predictor of developing burnout ${ }^{[14]}$. Good social support and other organizational factors such as good feedback and leadership are correlated with low levels of burnout in work environments ${ }^{[15]}$. Similarly, medical students with higher levels of social support are less likely to have burnout symptoms [16], Although psychological literatures have confirmed that social support could reduce burnout through resilience ${ }^{[17,18]}$, it is still unknown whether it would alleviate learning burnout in medical students and its alleviation mechanism.

Medical students are often exposed to academic pressure and a competitive environment which could cause the onset of learning burnout ${ }^{[2,5,19]}$. In this study, we aimed to assess how the social support that medical students received reduces learning burnout caused by online learning in isolation during the COVID-19 epidemic from a single college in Wuhan. In addition, we set out to explore which characteristics of the social support are associated with learning burnout. Moreover, we aimed to determine the effect size of social support by controlling for several demographic and occupational predictors of learning burnout.

\section{Method}

\section{Data collection}

The target population consisted of medical students at the Tongji Medical College Huazhong University of Science and Technology. The electronic survey was anonymous and confidential, and it was distributed electronically by WeChat tools to all 2241 students who participated in online learning on April 2020 with the Wenjuanxing platform. The study was approved by the institutional review boards of the Tongji Medical College Huazhong University of Science and Technology. 


\section{Measures/Instruments}

The self-administered questionnaire could be divided into 3 parts.

\section{Part 1 Demographic and learning characteristics}

Part 1 consisted of demographic data, including age, gender, grade, residence, household income in 2019, whether to be a class leader during college, whether to receive a scholarship during college, and learning data, such as online learning time per day, average number of interactions with teachers or classmates per lesson.

\section{Part 2 Learning burnout}

We used the Learning Burnout Scale (LBS) to measure online learning burnout of undergraduate students. LBS was developed by Chinese researchers based on the version of $\mathrm{MBI}^{[20]}$. It consisted of 20 items covering three domains of burnout: dejection (8 items), improper behavior (6 items) and reduced personal accomplishment (6 items). Items were scored on a 5-point Likert scale ranging from 1 (totally disagree) to 5 (totally agree). Additionally, we modified the expression of some items according to the characteristics of online learning (for example, changing the following "I felt exhausted after learning for a whole day" to "I felt exhausted after online learning for a whole day"). Learning burnout was defined as mean item score $\geq 3$. The Cronbach's alpha achieved $0.907,0.828,0.787$, and 0.776 in LBS and its dimensions of dejection, improper behavior, and reduced personal accomplishment, respectively.

\section{Part 3 Social support}

Social support was assessed using the SSRS, which was developed by a Chinese researcher ${ }^{[21]}$. This widely utilized instrument includes three measurable dimensions of social support: subjective support (4 questions) and utilization of support (3 questions) and objective support (3 questions). Each response of subjective support and utilization of support is rated on a 4-point Likert scale. Objective support is measured according to the number of social support source. Researchers usually use the total score to assess social support and have demonstrated that SSRS had good predictive validity and internal consistency among Chinese medical students ${ }^{[21,22]}$. Besides, we calculated the Cronbach's alpha (0.678) to measure the internal consistency of SSRS in the present study.

\section{Statistical analysis}

All analyses were performed with SPSS version 21 (IBM Corp., Armonk, NY, USA).rcentages, and continuous variables as mean \pm standard deviation. Categorical variables were compared with chi-square tests, and continuous variables with Student's t-tests or analysis of variance. Multivariate logistic regression analysis was conducted to evaluate the association between social support (SSRS scores) and learning burnout, adjusted for factors that were independently related to learning burnout. A p-value of 0.05 (two-tailed) was considered to be statistically significant. 


\section{Results}

\section{Descriptive statistics of demographics and learning burnout}

From the 2214 students who were invited to participate, a total of 684 responses were received giving an overall response rate of $30.9 \%$. Since medical students should study and live in the main campus in their first year, students in our college were mainly sophomores and juniors, accounting for $45.39 \%$ and $34.26 \%$. The median age of the students was 20 years (range $17-24$ ). Over fifty percent of respondents were female. The numbers (percentages) of municipalities or provincial capitals, prefecture-level cities, county-level cities, and town or rural areas were 132(19.33\%), 167 (24.45\%), 199(29.14\%), and 185 (27.09\%), respectively. More than three-fifths (63.69\%) of the students' family income in 2019 bellows 14, $150 \$$. A total of 367 respondents served as class cadres and 388 respondents received scholarships during college. Our results showed that $315(46.12 \%)$ students displayed evidence of learning burnout, with 370 (54.1\%) reporting high dejection, 329 (48.1\%) reporting high improper behavior, and 295 (43.19\%) reporting high reduced personal accomplishment during the online study. Table 1 shows the demographic characteristics and learning burnout of the responding students.

Table 1. Demographic Characteristics and learning burnout of respondents

\section{Analysis of differences in variables according to demographic and learning characteristics}

Table 2 displays differences of the numbers and percentages of learning burnout among demographic and online learning feature groups. We found that age $(p=0.084)$, gender $(p=0.670)$, residence $(p=$ $0.107)$ and whether to be a class leader $(p=0.466)$ or obtain a scholarship $(p=0.123)$ were not related to learning burnout. However, we found grade $(p=0.002)$, total household income in $2019(p=0.008)$, learning time $(p<0.001)$ and communication times $(p<0.001)$ were relevant to learning burnout. Considering that the residence was related to the two dimensions of learning burnout (Improper behavior, $\chi^{2}=9.624 p=0.047$; Reduced personal accomplishment, $\chi^{2}=14.336 p=0.006$ ), we took it together with grade as confounding variables and incorporated them into in a multivariate regression analysis. We found an inverse relationship between the mean SSRS score and the risk of learning burnout (adjusted odds ratio, 0.93 for 1-point decrease in total SSRS score; $95 \% \mathrm{Cl}, 0.90-0.96 ; \mathrm{p}<0.001$ ).

Table 2. Differences in learning burnout by demographic and online learning characteristics.

\section{Analysis of the relationship between SSRS and learning burnout}

We used student's t tests and binary regression to identify if students' social support was affected by various learning burnout symptoms. Results revealed that regardless of whether students presented learning burnout, SSRS scores were all decreased if they exhibited the corresponding symptoms (Table 3). Besides, we found a negatively relationship between social support and learning burnout $(r=-0.240$; $P<0.01)$. Students, who exhibited a syndrome of learning burnout, had a lower score on subjective 
support and utilization of support $(t=4.510, P<0.01 ; t=4.158, P<0.01)$, but no difference in objective support $(t=1.128, P>0.05)$. (Table 4)

Table 3. Differences in students' SSRS scores by learning burnout, dejection, improper behavior and reduced personal accomplishment groups

Table 4 Differences in the subscale of social support scores according to the presence of learning burnout syndrome

\section{Discussion}

\section{Main findings}

In this study, we examined the association between social support and learning burnout. We found that even after adjusting for the grade and residence, there was a significant and relevant association between the social support and learning burnout in our sample of Tongji medical college.

\section{Learning burnout prevalence}

In our study, we found that an average of $45.9 \%$ students had symptoms suggestive of learning burnout. The result was much higher than the rates of $21.76 \%$ and $36.46 \%$ of Chinese medical students, that were described by Tang et ${ }^{[23]}$ and Yang ${ }^{[24]}$, who used the same instrument and criteria. Students in Kingdom of Saudi Arabia had a moderate to high level of stress at the start of the COVID-19 outbreak ${ }^{[25]}$. Our students mainly came from Hubei province, which was the earliest and the worst area affected by COVID19 in China. Therefore, they may have more physical and psychological stress, which was related to high burnout scores, during this period of home isolation.

\section{Environmental factors associated with learning burnout}

In this study, we found that the prevalence of learning burnout was higher for students in more advanced years. The result was in line with previous research ${ }^{[5]}$. This may be due to the pressure of senior students facing employment or internships ${ }^{[26-28]}$. Besides, we calculated that the burnout rate of graduation students $(63.6 \%)$ was much higher than that of non-graduation students $(45.2 \%)$. Studies indicated that medical graduates faced more pressure, which involved in high burnout, than non-graduates ${ }^{[29,30]}$. The possible reason could be that the uncertainty about the pandemic effect may have increased their worry about graduating, finding a job or enrolling in further study [31].

We found that environmental factors, especially those related to the economy, were closely related to learning burnout. Besides, whilst there was no significant difference in burnout rates of medical students from different residences, medical students from rural areas had a significantly higher rate than those from cities in improper behavior or reduced personal accomplishment dimension. The economic pressure may be considered to facilitate the development of burnout ${ }^{[32,33]}$. Students with family difficulties or in 
rural areas had to face extra pressure of the economy from their families. This situation may give rise to more learning burnout. Besides, our results showed that the higher the learning time and frequency of communication, the lower the learning burnout. This phenomenon suggested that communicating with teachers or students may reduce students' learning burnout and increase the learning time. The two factors were all closely related to social support, especially the subjective support and utilization of support.

\section{The protective effect of social support on learning burnout}

Social support have a protective effect for burnout symptom in medical students ${ }^{[34]}$. We found a similar effect of social support on learning burnout, but the subjective support and utilization of support have a greater impact on learning burnout. A meta-analysis also reported that seeking social support from friends or family members was already found to be correlated with burnout in a work setting ${ }^{[35]}$. Lazarus's stress and coping theory holds that active communication was an effective way to relieve stress. Our results also demonstrated that subjective support and utilization of support could reduce learning burnout by communicating with relatives or friends. Social support provides a buffering effect

against stress in that an individual who has more social support is also more resilient to stress ${ }^{[36]}$. Besides, Thoits argued that social support served to regulate the stress itself and also provided a coping context, which could help the individual cope with stress or buffer the person against the demands ${ }^{\text {[37] }}$. This prompted us to further explore what social support environment and other mechanisms of social support to alleviate learning burnout.

\section{Limitations}

The current study also has some limitations. First, although the response rate of $30.9 \%$ was relatively low, we collected 684 samples that exceed those of studies with higher response rates. Second, we measured students' burnout with LBS instead of MBI, Considering the differences in culture context, this may set obstacles comparing with peers over the world. However, this scale had been widely used in China. Moreover, our sample was drawn from a single school, and we should acknowledge that our results may be less generalizable to other schools and countries.

\section{Conclusion}

We found a high prevalence of learning burnout among students participating in online learning during the COVID-19 epidemic period. Students' grade, family income, learning time and number of interactions were all closely associated with learning burnout. Social support, especially subjective support and utilization of support provided a buffering effect against stress faced by medical students and hence the possibility of their developing learning burnout. Considering the harmfulness of learning burnout, it is necessary to further look for other risk factors of learning burnout. Moreover, further research is also needed to identify other mechanisms of social support to relieve learning burnout in medical students. 


\section{Abbreviations}

COVID-19: Corona Virus Disease 2019; LBS: Learning Burnout Scale; MBI: Maslach Burnout Inventory; SSRS: Social Support Rating Scale.

\section{Declarations}

\section{Funding}

This work was supported by the Huazhong University of Science and Technology Teaching Research Project and 'Double First Class' International Cooperation Project.

\section{Availability of data and materials}

Data generated or analyzed during this study are included in this published article.

\section{Authors' contributions}

ZJY designed, analyzed, and contributed in collecting the data, interpreting the results, and writing the draft manuscript. FZC contributed in guiding research design and revising the manuscript. ST and XM contributed in collecting and organizing the data. All authors read and approved the final manuscript.

\section{Author information}

${ }^{1}$ School of Medicine and Health Mangement, Tongji Medical College, Huazhong University of Science and Technology, No. 13 Hangkong Road, Qiaokou Region, Wuhan, China. ${ }^{2}$ Medical academic affairs office, Tongji Medical College, Huazhong University of Science and Technology, No. 13 Hangkong Road, Qiaokou Region, Wuhan, China. ${ }^{*}$ corresponding author

\section{Ethics approval and consent to participate}

This study was carried out according to the ethical principles for medical research involving human subjects of the WMA Declaration of Helsinki. No individual data were collected, anonymity was guaranteed, participation was voluntary, and informed consent was obtained. The ethical board of Tongji Medical College determined that the study was exempt from formal ethical review.

\section{Acknowledgments}

The authors would like to thank the faculties who assisted with the data collection for this questionnaire in Tongji Medical College. We would also like to thank the students for their time and effort in completing this questionnaire. We would like to thank WenJuanXing for providing us with such a platform to complete the questionnaire survey.

\section{Competing interests}


The authors declare that they have no competing interests.

Consent for publication

Not applicable.

\section{References}

1. J C, Y ZY, J W, H SY: Academic burnout and depression of Chinese medical students in the preclinical years: the buffering hypothesis of resilience and social support. Psychology, health \& medicine 2019.

2. Pagnin D, De Queiroz V, De Oliveira FM, Gonzalez NV, Salgado AE, Cordeiro EOB, Lodi CS, Melo RM: Burnout and career choice motivation in medical students. Medical teacher 2013, 35(5):388-394.

3. Schaufeli WB, Martinez IM, Pinto AM, Salanova M, Bakker AB: Burnout and Engagement in University Students: A Cross-National Study. Journal of Cross-Cultural Psychology 2002, 33(5):464-481.

4. Salmela-Aro K, Savolainen H, Holopainen L: Depressive Symptoms and School Burnout During Adolescence: Evidence from Two Cross-lagged Longitudinal Studies. Journal of Youth and Adolescence 2009, 38(10).

5. Chunming WM, Harrison R, Maclntyre R, Travaglia J, Balasooriya C: Burnout in medical students: a systematic review of experiences in Chinese medical schools. BMC Med Educ 2017, 17(1):217.

6. Paro HB, Silveira PS, Perotta B, Gannam S, Enns SC, Giaxa RR, Bonito RF, Martins MA, Tempski PZ: Empathy among medical students: is there a relation with quality of life and burnout? Plos One 2014, 9(4):e94133.

7. Dyrbye LN, Massie FJ, Eacker A, Harper W, Power D, Durning SJ, Thomas MR, Moutier C, Satele D, Sloan $\mathrm{J}$ et al: Relationship between burnout and professional conduct and attitudes among US medical students. Jama 2010, 304(11):1173-1180.

8. Brazeau CM, Schroeder R, Rovi S, Boyd L: Relationships between medical student burnout, empathy, and professionalism climate. Acad Med 2010, 85(10 Suppl):S33-36.

9. Wallace JE, Lemaire JB, Ghali WA: Physician wellness: a missing quality indicator. The Lancet 2009, 374(9702).

10. Fahrenkopf AM, Sectish TC, Barger LK, Sharek PJ, Lewin D, Chiang VW, Edwards S, Wiedermann BL, Landrigan CP: Rates of medication errors among depressed and burnt out residents: prospective cohort study. BMJ (Clinical research ed) 2008, 336(7642):488-491.

11. PY P: A Study on the Relationships of Mental Stress, Social Support and Learning Burnout of College Adult Undergraduate Students. Master. Guangzhou University; 2011.

12. YD J: Study on the Relationship of Social Support, Psychological Capital and Learning Burnout of College Students. Master. Harbin Engineering Univerty; 2014.

13. Cañadas-De la Fuente GA, Vargas C, San Luis C, García I, Cañadas GR, De la Fuente El: Risk factors and prevalence of burnout syndrome in the nursing profession. International journal of nursing 
studies 2015, 52(1):240-249.

14. García-Izquierdo M, Ríos-Rísquez MI: The relationship between psychosocial job stress and burnout in emergency departments: an exploratory study. Nursing outlook 2012, 60(5):322-329.

15. Melchior ME, van den Berg AA, Halfens R, Huyer Abu-Saad H, Philipsen H, Gassman P: Burnout and the work environment of nurses in psychiatric long-stay care settings. Social psychiatry and psychiatric epidemiology 1997, 32(3):158-164.

16. T PJ, M G-DS, J TB, A vdHFMM, M vdWHB, M H-WJEH: Burnout in medical residents: a review. Medical education 2007, 41(8).

17. A KL, W KD, A FJ, M KT, A AG: Resilience-recovery factors in post-traumatic stress disorder among female and male Vietnam veterans: hardiness, postwar social support, and additional stressful life events. Journal of personality and social psychology 1998, 74(2).

18. Amanda $\mathrm{H}$, Anna $\mathrm{S}$, Andrea S: Towards an understanding of resilience and its relevance to medical training. Medical education 2012, 46(4).

19. Katariina S-A, Katja U: School burnout and engagement in the context of demands-resources model. The British journal of educational psychology 2014, 84(Pt 1).

20. Lian R YLX, Wu L H: The relationship between college students' professional commitment and learning burnout and the development of the scale. Acta Psychologica Sinica 2005(05):632-636.

21. Xiao SY: The theoretical basis and application of social support scale. Journal of Clinical 1994(02):98-100.

22. Zhang J R HY: The correlation between social support, coping style and subjective well-being of college students. Chinese Journal of Clinical Psychology 2007(06):629-631.

23. Tang T LSWYJ, Huang D Y: Analysis of the status quo and influencing factors of medical students' burnout in Jishou University. Journal of Social Medicine 2019, 17(06):328-331.

24. Yang DL: The study on group intervention in learning burnout of medical students. Master. ShanXi Medical University; 2011.

25. AlAteeq DA, Aljhani S, AlEesa D: Perceived stress among students in virtual classrooms during the COVID-19 outbreak in KSA. Journal of Taibah University Medical Sciences 2020.

26. M WS, G DM, C TC, J AB: Burnout and psychiatric morbidity in new medical graduates. The Medical journal of Australia 2004, 181(7).

27. M D, N J, B R: Performance-based self-esteem and burnout in a cross-sectional study of medical students. Medical teacher 2007, 29(1).

28. MD DLN, MD TMR, L. HJ, MD LKL, MS NPJ, PhD SJA, MD STD: Personal Life Events and Medical Student Burnout: A Multicenter Study. Academic Medicine 2006, 81(4).

29. Lal A, Tharyan A, Tharyan P: The prevalence, determinants and the role of empathy and religious or spiritual beliefs on job stress, job satisfaction, coping, burnout, and mental health in medical and surgical faculty of a teaching hospital: A cross-sectional survey. La revue de médecine interne 2020, 41(4). 
30. Iorga M, Dondas C, Zugun-Eloae C: Depressed as Freshmen, Stressed as Seniors: The Relationship between Depression, Perceived Stress and Academic Results among Medical Students. Behavioral sciences (Basel, Switzerland) 2018, 8(8).

31. Tang W, Hu T, Hu B, Jin C, Wang G, Xie C, Chen S, Xu J: Prevalence and correlates of PTSD and depressive symptoms one month after the outbreak of the COVID-19 epidemic in a sample of homequarantined Chinese university students. J Affect Disord 2020, 274:1-7.

32. Manzano-García G, Montañés P, Megías JL: Perception of economic crisis among Spanish nursing students: Its relation to burnout and engagement. Nurse education today 2017, 52:116-120.

33. Marisa P, T ESR, Michael S: Quality of Life and Burnout Rates Across Surgical Specialties: A Systematic Review. JAMA surgery 2016, 151(10).

34. Kim B, Jee S, Lee J, An S, Lee SM: Relationships between social support and student burnout: A meta-analytic approach. Stress and Health 2018, 34(1).

35. B HJR: Sources of social support and burnout: a meta-analytic test of the conservation of resources model. The Journal of applied psychology 2006, 91(5).

36. Cassidy T: Stress, cognition and health: Routledge; 1999.

37. A TP: Social support as coping assistance. Journal of consulting and clinical psychology 1986, $54(4)$.

\section{Tables}

Table 1. Demographic Characteristics and learning burnout of Respondents 


\begin{tabular}{lll}
\hline Variables & Items & $\mathrm{N}(\%)$ \\
\hline Grade & second & $310(45.39)$ \\
& third & $234(34.26)$ \\
& forth & $128(18.74)$ \\
Median age, years (range) & fifth & $11(1.61)$ \\
Gender & & $20117-24 \square$ \\
Area of residence & male & $290(42.46)$ \\
& female & $393(57.54)$ \\
& municipalities/provincial capitals & $132(19.33)$ \\
& prefecture-level city & $167(24.45)$ \\
Total household income in 2019 & county-level cities & $199(29.14)$ \\
& town & $65(9.52)$ \\
Improper behavior & rural areas & $120(17.57)$ \\
& $50,000 ¥$ and bellow & $197(28.84)$ \\
Reduced personal accomplishment & $50,000 ¥-100,000 ¥$ & $238(34.85)$ \\
& $110,000 ¥-150,000 ¥$ & $111(16.25)$ \\
Whether to be a class leader during college & $150,000 ¥-200,000 ¥$ & $68(9.96)$ \\
Whether to obtain a scholarship during college & no & $69(10.1)$ \\
& no & $316(46.27)$ \\
& yes & $367(53.73)$ \\
& no & $295(43.19)$ \\
& yes & $388(56.81)$ \\
& no & $368(53.88)$ \\
& yes & $315(46.12)$ \\
& no & $313(45.83)$ \\
& yes & $370(54.17)$ \\
& no & $355(51.98)$ \\
& yes & $328(48.02)$ \\
& & $383(56.08)$ \\
& & $300(43.92)$ \\
\hline
\end{tabular}

Table 2. Differences in learning burnout and its subscales by demographic and online learning characteristics. 


\begin{tabular}{|c|c|c|}
\hline \multirow[t]{2}{*}{ Variables } & \multicolumn{2}{|c|}{ Learning burnout } \\
\hline & $\mathrm{N}(\%)$ & $\chi^{2}(p)$ \\
\hline \multicolumn{3}{|l|}{ Grade } \\
\hline second & 123(39.68) & 14.607 \\
\hline third & $111(47.44)$ & $(0.002)$ \\
\hline forth & $73(57.03)$ & \\
\hline fifth & $8(72.73)$ & \\
\hline \multicolumn{3}{|l|}{ Age } \\
\hline 18 and bellow & $4(28.57)$ & 6.661 \\
\hline $19-20$ & $163(42.78)$ & $(0.084)$ \\
\hline $21-22$ & $132(51.36)$ & \\
\hline 23 and above & $16(51.61)$ & \\
\hline \multicolumn{3}{|l|}{ Gender } \\
\hline Male & $131(45.17)$ & 0.182 \\
\hline Female & $184(46.82)$ & $(0.67)$ \\
\hline \multicolumn{3}{|l|}{ Residence } \\
\hline Municipalities/ & $51(38.64)$ & 7.603 \\
\hline \multicolumn{3}{|l|}{ provincial capitals } \\
\hline prefecture-level city & $77(46.11)$ & $(0.107)$ \\
\hline county-level cities & $91(45.73)$ & \\
\hline town & $29(44.62)$ & \\
\hline rural areas & $67(55.83)$ & \\
\hline \multicolumn{3}{|l|}{ Family income in 2019} \\
\hline $7,075 \$$ and bellow & $110(55.84)$ & 13.9 \\
\hline $7,075 \$$ to $14,150 \$$ & $110(46.22)$ & $(0.008)$ \\
\hline $14,150 \$$ to $21,225 \$$ & $41(36.94)$ & \\
\hline $21,225 \$$ to $28,300 \$$ & $28(41.18)$ & \\
\hline $28,300 \$$ and above & $26(37.68)$ & \\
\hline \multicolumn{3}{|c|}{ Whether to be a class leader during college } \\
\hline no & $141(44.62)$ & 0.532 \\
\hline yes & $174(47.41)$ & $(0.466)$ \\
\hline \multicolumn{3}{|c|}{ Whether to obtain a scholarship during college } \\
\hline no & 146(49.49) & 2.375 \\
\hline yes & $169(43.56)$ & $(0.123)$ \\
\hline \multicolumn{3}{|c|}{ Online learning time per day } \\
\hline $2 \mathrm{~h}$ and bellow & $58(92.06)$ & 107.699 \\
\hline $2-4 \mathrm{~h}$ & 126(59.15) & $(<0.001)$ \\
\hline $4-6 \mathrm{~h}$ & $91(37.92)$ & \\
\hline $6-8 \mathrm{~h}$ & $28(24.78)$ & \\
\hline $8 \mathrm{~h}$ and above & $12(22.22)$ & \\
\hline \multicolumn{3}{|c|}{ Average number of interactions with teachers or classmates per lesso } \\
\hline 2 and bellow & 203(57.02) & 44.915 \\
\hline $2-4$ & $86(40.19)$ & $(<0.001)$ \\
\hline $4-6$ & $16(23.53)$ & \\
\hline $6-8$ & $7(26.92)$ & \\
\hline 8 and above & $3(15.79)$ & \\
\hline
\end{tabular}


Table 3. Differences in students' SSRS scores by learning burnout, dejection, improper behavior and reduced personal accomplishment groups

\begin{tabular}{lllcc}
\hline Scale & Item & Mean $\pm \mathrm{sd}$ & $\mathrm{t}$ & $\mathrm{p}$ \\
\hline Learning burnout & Not burnout & $27.86 \pm 4.60$ & 4.370 & $<0.001$ \\
& Burnout & $26.33 \pm 4.53$ & & \\
Dejection & Not dejected & $27.99 \pm 4.52$ & 4.416 & $<0.001$ \\
& Dejected & $26.45 \pm 4.60$ & & \\
Improper behavior & Not have & $27.93 \pm 4.63$ & 4.602 & $<0.001$ \\
& Have & $26.32 \pm 4.48$ & & \\
Reduced personal accomplishment & Not competent & $27.76 \pm 4.49$ & 3.917 & $<0.001$ \\
& Competent & $26.38 \pm 4.69$ & & \\
\hline
\end{tabular}

Table 4 Differences in the subscale of social support scores according to the presence of learning burnout syndrome

\begin{tabular}{|c|c|c|c|c|}
\hline \multirow[t]{2}{*}{ Scale } & Learning burnout & Without learning burnout & \multirow[t]{2}{*}{$\mathrm{t}$} & \multirow[t]{2}{*}{$\mathrm{p}$} \\
\hline & Mean \pm sd & Mean \pm sd & & \\
\hline Subjective support & $11.44 \pm 2.05$ & $12.14 \pm 1.98$ & 4.510 & $<0.001$ \\
\hline Objective support & $8.12 \pm 1.93$ & $8.29 \pm 2.05$ & 1.128 & 0.260 \\
\hline Utilization of support & $6.77 \pm 2.02$ & $7.43 \pm 2.12$ & 4.158 & $<0.001$ \\
\hline
\end{tabular}

\section{Supplementary Files}

This is a list of supplementary files associated with this preprint. Click to download.

- originaldata.xlsx 